\title{
EPISTEMOLOGÍA FEMINISTA DE LA CONCILIACIÓN: UNA PROPUESTA PRÁCTICA PARA MIRADAS Y MUJERES EN TRANSICIÓN
}

\section{CONCILIATORY FEMINIST EPISTEMOLOGY: A PRACTICAL PROPOSAL FOR LOOKS AND WOMEN IN TRANSITION}

\author{
Laura MARTÍNEZ-JIMÉNEZ
}

\author{
Author / Autora: \\ Laura Martínez-Jiménez \\ Universidad Rey Juan Carlos \\ Madrid, España \\ laura.martinezj@urjc.es \\ https://orcid.org/0000-0002-1678-0983 \\ Submitted / Recibido: 14/07/2021 \\ Accepted / Aceptado: 13/10/2021
}

To cite this article / Para citar este artículo: Martínez-Jiménez, L. (2022). Epistemología feminista de la conciliación: una propuesta práctica para miradas y mujeres en transición. Feminismo/s, 39, 181-210. https://doi. org/10.14198/fem.2022.39.07

Licence / Licencia:

This work is licensed under a Creative Commons Attribution 4.0 International.

\section{(c) (1)}

(C) Laura Martínez-Jiménez

\section{Resumen}

Este trabajo reclama la pertinencia de una práctica epistemológica feminista de la conciliación, capaz de aunar sensibilidades y quehaceres modernos y posmodernos. Esta mirada conciliadora permite abordar en su amplia complejidad las relaciones entre las dimensiones macroestructurales, mesoinstitucionales y microencarnadas de las vidas de las mujeres, así como transitar los actuales debates en torno a la (in)validez de las categorías de género y mujer. Para ello, se toma como referencia de partida la Teoría del Punto de Vista de Sandra Harding, paradigma de la nueva epistemología feminista y foco de discrepancias científicas y políticas entre los propios feminismos. A partir de la propuesta de Harding, se analizan críticamente las tensiones feministas que esta teoría plantea y que apuntan hacia ciertos riesgos esencialistas y universalistas y, al mismo tiempo, hacia posibles derivas individualistas o relativistas. Se sugiere entonces el encuentro de esta Teoría del Punto de Vista con un enfoque posmoderno que la complejice y actualice al calor de las actuales discusiones feministas. Ya desde este punto de vista posmoderno, se plantean una serie de enmiendas para afinar la mirada 
epistemológica feminista: enfrentar el relato único sobre las mujeres a partir de sus diversas experiencias; explorar las distintas experiencias encarnadas para conectarlas y analizarlas en contexto, esto es, sujetas a las relaciones estructurales de poder; sustituir el privilegio epistémico automático por una preferencia crítica y argumentada por las experiencias y relatos de las mujeres; y, finalmente, poner en valor las experiencias y relatos de la propia investigadora para orientar el estudio y, también, exponer los privilegios y limitaciones de su punto de vista.

Palabras clave: epistemología feminista; género; teoría del punto de vista; teoría posmoderna.

\begin{abstract}
This essay claims the relevance of a feminist epistemological practice of conciliation, capable of bringing together modern and postmodern sensibilities and works. This conciliatory gaze makes it possible to address the relationships between the macro-structural, meso-institutional and micro-incarnate dimensions of women's lives in their broad complexity, as well as to go through the current debates on the (in)validity of the categories of gender and woman. To this end, Sandra Harding's Standpoint Theory, a paradigm of the new feminist epistemology and the focus of scientific and political discrepancies between feminisms themselves, is taken as a starting point. Based on Harding's proposal, a critical analysis is made of the feminist tensions that this theory raises. These tensions point to certain essentialist and universalist risks and, at the same time, to possible individualist or relativist drifts. It is then suggested that this Standpoint Theory should meet with a postmodern approach that would make it more complex and update it in the eyes of current feminist discussions. From this postmodern standpoint, a series of amendments are proposed to refine the feminist epistemological gaze: to confront the single narrative about women from their diverse experiences; to explore the different embodied experiences to connect and analyse them in context, that is, subjected to structural power relations; to replace automatic epistemic privilege with a critical and argued preference for women's experiences and narratives; and, finally, to value the experiences and stories of the researcher to guide the study and, also, to expose the privileges and limitations of their own point of view.
\end{abstract}

Keywords: feminist epistemology; gender; standpoint theory; postmodern theory. 


\section{INTRODUCCIÓN: EPISTEMOLOGÍA FEMINISTA PARA MIRAR Y VIVIR BIEN}

Parapetado tras las nociones de universalidad, objetividad, neutralidad y racionalidad (Blázquez, 2011, 2012; Harding, 2002) y deliberadamente ajeno a la emocionalidad (Hernando, 2012), el quehacer tradicional de la ciencia occidental viene privilegiando la visión y experiencia del que Amaia PérezOrozco (2014, p. 39), siguiendo a María José Capellín, denomina «sujeto BBVAh»: «el sujeto blanco, burgués, varón, adulto, con una funcionalidad normativa [y] heterosexual». Un sujeto que encarna paradigmáticamente la independencia, la racionalidad y el hiperindividualismo del homo economicus adalid del capitalismo neoliberal (Gálvez, 2017; Gill y Roberts, 2011; Pérez-Orozco, 2014) y que Almudena Hernando (2012, pp. 147-148) equipara a la «individualidad dependiente» propia de la masculinidad hegemónica: un tipo de identidad que se relaciona con el mundo desde «la fantasía de la individualidad»-esto es, desde su pretendida autosuficiencia- mientras invisibiliza o niega el consuelo y el privilegio que le proporcionan las relaciones de desigualdad que ejerce particularmente sobre las mujeres y de las cuales (de sus cuidados y trabajos), paradójicamente, depende ${ }^{1}$. La centralidad de este sujeto privilegiado como estándar también científico ha obscurecido las transformaciones sociohistóricas de las normas, identidades y valores de género (Gill y Roberts, 2011), ya que si hay algo que no es este homo economicus es, precisamente, una mujer (Gálvez, 2017). Como sostiene Sandra Harding (2002, p. 21), la prerrogativa de esta perspectiva androcéntrica en la determinación de las cuestiones científicas no genera sino «visiones parciales y hasta perversas» de la realidad social, de tal forma que la epistemología convencional -la «epistemología heteropatriarcal» (Pérez-Orozco, 2014, p. 81) - se demostraría alevosa, irresponsable o inercialmente inepta para comprender y explicar los lugares y las prácticas sociales de las mujeres (Blázquez, 2011; Harding, 2002, 2004).

1. Al escribir sobre las mujeres como sujeto colectivo indeterminado, sin más apellidos, este trabajo no renuncia a la (aún) necesidad política y teórica de hablar sobre/para nosotras, pero lo hace sometiéndola al cuestionamiento crítico al que invitan la interseccionalidad y diversidad de las experiencias de feminidad y las propias diferencias intragénero. 
Esta impericia de la epistemología convencional(mente) androcéntrica se condensa en lo que podríamos calificar como el diamante de discriminación de las mujeres en el conocimiento científico. Por una parte, las mujeres -así como sus sensibilidades y saberes derivados de su socialización diferenciadason expulsadas de la producción y la autoridad científicas (Blázquez, 2012; Harding, 2002, 2004). Por otro lado, la teorización androcéntrica menoscaba la visibilidad, validez e importancia de las prácticas y los intereses de las mujeres (Blázquez, 2012), ejerciendo incluso en contra de sus deseos y necesidades y a favor de una masculinidad presumiblemente occidental y burguesa (Harding, 1996, 2004). Además, esta forma de hacer ciencia menosprecia las implicaciones de género -también de clase, raza, sexualidad, diversidad funcional...- de las relaciones de poder, produciendo pues un cuerpo de conocimientos fundamental para la ejecución y legitimación de «proyectos sociales sexistas, racistas, homofóbicos y clasistas» (Harding, 1996, p. 20) que, mediante teorías que subestiman, incapacitan y/o distorsionan a las mujeres, reproducen las desigualdades de género (Blázquez, 2012). Por tanto (y por último), la ciencia tradicional habría venido normalizando y normativizando determinadas representaciones y sentidos de la feminidad (Biglia, 2015; Blázquez, 2011), interpretados por las investigadoras feministas como un deseo o fantasía de la ciencia androcéntrica de confeccionar un ideal de mujer adecuado y, sobre todo, útil, desde el cual «apaciguarlas, controlarlas, explotarlas o manipularlas» (Harding, 2002, p. 24) y situarlas en la alteridad u otredad respecto a la masculinidad emblemática. Esta lógica dicotómica -también binaria y heteronormativa- aspiraría a dominar la explicación del mundo, y el mundo mismo, desde el ejercicio de los privilegios de unos sujetos masculinos-occidentales-burgueses sobre las mujeres (Blázquez, 2012; Harding, 1996), e incluso de ciertas mujeres sobre sus otras.

Frente al discurso y las prácticas de la ciencia androcéntrica, las filósofas, epistemólogas e investigadoras feministas vendrían especulando -no sin disenso, especialmente entre los marcos modernos y posmodernos de pensamiento y acción (ver Bartra, 2002; Biglia, 2015; Harding, 2002; Walby, 2001) - sobre métodos, metodologías o epistemologías alternativas y estrictamente feministas (Bartra, 2002; Harding, 2002; Hollows, 2000), a través de las cuales no solo comprender las vidas en sociedad (Blázquez, 2012), sino también aspirar a la «acción política» (Hollows, 2000, p. 19). Precisamente, 
desde las fricciones y desacuerdos entretejidos en el seno de los feminismos (sin negarlos ni romantizar su aún tenso diálogo), este trabajo quiere reclamar la pertinencia de una práctica epistemológica feminista de la conciliación, capaz de aunar sensibilidades y quehaceres del feminismo heredero de la Modernidad (aspirante a generar teorías, relatos y categorías críticas y fuertes que nos sirvieran a todas para enfrentar la materialidad del patriarcado) y aquellos feminismos posmodernos alumbrados por las políticas de lo personal-situado y la interseccionalidad y por el giro académico posestructuralista hacia lo discursivo ${ }^{2}$. Para ello, en el siguiente apartado se toma como referencia de partida la Teoría del Punto de Vista de Sandra Harding, paradigma de la nueva epistemología feminista y foco de discrepancias científicas y políticas entre los propios feminismos. A partir de la propuesta de Harding, en el tercer apartado se analizan críticamente las tensiones feministas que esta teoría plantea y que apuntan hacia ciertos riesgos esencialistas y universalistas y, al mismo tiempo, hacia posibles derivas individualistas o relativistas. A continuación, se defiende el interés del encuentro de esta Teoría del Punto de Vista con un enfoque posmoderno que la complejice y actualice al calor de las actuales discusiones feministas. Finalmente, ya desde un punto de vista posmoderno, se plantean una serie de enmiendas para afinar la mirada epistemológica feminista partiendo de la responsabilidad autorreflexiva de la propia investigadora.

\section{LA TEORÍA FEMINISTA DEL PUNTO DE VISTA Y SUS TENSIONES: ESENCIALISMO, UNIVERSALISMO, REPRESENTATIVIDAD}

Siguiendo el espíritu y los objetivos de estos saberes de acción feminista, la propuesta epistemológica más sugerente parece formularla la Teoría del Punto de Vista (Standpoint Theory), desarrollada y defendida por autoras como Sandra Harding (1996, 2004, 2012), Nancy Hartsock (1997) o Patricia Hill Collins (1997). Desde su radical interés en las relaciones entre poder y conocimiento (Harding, 2004; Hill Collins, 1997), esta diversa estrategia

2. Para profundizar en los diálogos pendientes entre los feminismos herederos del pensamiento y el proyecto modernizador y los feminismos posmodernos, se recomienda la lectura de Nicholson (1992). 
epistemológica-que renombraremos en este trabajo como Teorías Feministas de los Puntos de Vista (en adelante, TTFFPPV) para dar cuenta así de dicha diversidad-aspira a modificar las situaciones subalternas de las mujeres y mejorar sus condiciones de vida produciendo conocimiento con y para ellas a partir de sus propias voces y experiencias. De tal forma, en esta propuesta, ciencia y política concurren y no ocurren la una sin la otra (Harding, 2004).

Con todo, la propuesta feminista del punto de vista es reconocida por la propia Harding (1996, pp. 123, 142-143) como una «meditación aún en transición» que, por tanto, estimula ciertas «ambivalencias». Un proyecto, asimismo, de límites cada vez más difusos, líquidos y elásticos respecto a las proposiciones epistemológicas empirista y posmoderna (Blázquez, 2011, 2012; Harding, 1996; Hekman, 1997). Y objeto, además, de no pocas polémicas y controversias (Harding, 2004, 2012; Hirschmann, 1998), que cuestionan su interés y cientificidad (Hekman, 1997; Walby, 2001) y, por extensión, su potencial político transformador (Harding, 2004).

Por ello, en las páginas que vienen no solo se argumentan los motivos por los cuales esta investigación se inscribe críticamente en la epistemología feminista de los puntos de vista. Además, se expone la responsabilidad de situarse dentro de la problemática que implica una teoría plural, viva y en transición y, por tanto, potencialmente conflictiva como esta, reconociendo, como hace Harding (1996, p. 143), que «las ambivalencias en el feminismo constituyen guías provechosas» para explicar un mundo igualmente tenso, contradictorio y ambivalente. Así, las tensiones, contradicciones y ambivalencias de las TTFFPPV ofrecen la oportunidad de complejizar dichas teorías y fundamentar una aproximación crítica a la epistemología feminista posmoderna capaz de enfrentar las sospechas de implicaciones más delicadas para los debates actuales, que no son sino aquellas relativas a la alargada sombra esencialista y universalizante que amenaza constantemente los estudios sobre identidades, mujeres y feminidad.

Lejos de defender una noción deliberadamente homogénea, naturalizada o absoluta de la feminidad, las investigadoras de las TTFFPPV suelen expresar la diversidad de las mujeres y las diferencias entre ellas mismas, sus condiciones de vida y sus experiencias (Harding, 1996, 2004; Smith, 1997). Con todo, las problemáticas sobre esencialismo, universalismo y representatividad resisten en el seno de la propuesta (Harding, 2004, 2012;

Feminismo/s 39, January 2022, 181-210 
Hartsock, 1997; Hekman, 1997; Hirschmann, 1998; Phipps, 2016; Walby, 2001). Como reconoce Harding (1996), la constante invocación de las experiencias y los puntos de vista de las mujeres, así como la utilización de la identidad de género como vector analítico, presentan ciertas complicaciones que ni siquiera sus intenciones emancipadoras o transformadoras pueden sortear, al igual que ocurre con las «diferencias esencialistas» que, a pesar de su dimensión crítica, entraña la reivindicación de las identidades sociales (Brah, 2011, p. 155). La instrumentalización subversiva de nociones clave y aún valiosas para el feminismo como mujer y feminidad, explica Harding (1996, pp. 149-151, 155), conservaría «residuos coloniales y patriarcales» en la medida en que también son artefactos fundamentales para el proyecto de dominación masculina que, además, entrañan riesgos de «ahistoricidad» y «universalizaciones excesivas». Asimismo, estas tensiones parecen inflamarse también desde el momento en el cual las investigaciones convierten un fenómeno social en un «problema para alguien» (Harding, 2002, pp. 20-21), en este caso para las mujeres y más concretamente, como destacan algunas autoras críticas, para un grupo privilegiado de ellas, cuyos intereses particulares, como denuncia bell hooks (2017), son legitimados como unitarios y representativos gracias, en parte, a la atención pública y mediática que reciben.

\section{1. ¿Un punto de vista único, compartido y, además, feminista?}

La patente pluralidad de feminismos, entendida como «estimulante» expresión de «las diferencias de percepción de las mujeres con respecto a quiénes somos y a la política adecuada para desenvolvernos en nuestras relaciones sociales cotidianas», pone de manifiesto la dificultad, e incluso, para muchas, la imposibilidad de establecer un único punto de vista epistemológico feminista, cuestionando así las supuestas «experiencias comunes» de las mujeres y, por extensión, la pretendida identidad compartida como fuerzas centrípetas que fundamentarían «las bases de una epistemología y una política [feministas] características» (Harding, 1996, p. 142). Por otra parte, debemos aclarar que no todos los puntos de vista de las mujeres pueden considerarse feministas per se. Es más, la propia Harding (2012, p. 51) reconoce que las narraciones y creencias de las mujeres son habitualmente contaminadas por 
«las representaciones distorsionadas de las relaciones sociales» que producen y popularizan los grupos dominantes a través de la hegemonía de sus discursos, llegando incluso a encarnar «subjetividades cómplices» (MartínezJiménez et al., 2018; Pérez-Orozco, 2014, p. 107). Recogiendo las palabras de Andrew Sayer (2009, p. 772), «es probable que el conocimiento que es subyugado lleve las marcas, incluidas las deficiencias, del conocimiento dominante». Por ello, tal y como hizo Nancy Hartsock (1997), debemos distinguir entre puntos de vista femenino(s) y feminista, con el afán de negar cualquier generación espontánea o natural de un punto de vista feminista o de un sujeto político colectivo. La diferencia entre ambas miradas la determinaría, creemos, lo que Stéphanie Genz (2009, p. 39) llama el ««nacimiento feminista» de cada una de las mujeres: un «acto de negación y trascendencia»», un reconocimiento del menoscabo para con su ser y finalmente «una voluntad de guerrear contra aquellas fracciones de la sociedad y de su ser que han socavado sus intentos de libertad».

Con todo, como matiza Charlotte Brunsdon (2007, pp. 486, 494-495), esto no quiere decir que la feminidad en sí misma implique un estado de «falsa consciencia», ni que la propia conciencia feminista ubique a sus iluminadas «por fuera» de la ideología. Quiere decir que el punto de vista feminista implica un esfuerzo crítico para mirarse a una misma, pero también comprometido, para mirarse junto a las demás, en relación a ellas y a un estado de cosas que sujeta las experiencias femeninas a las relaciones de poder (no solo generizadas); supone, así, «hablar» más allá de una misma, pero «aprendiendo primero a hablar por/de una misma». En palabras de Brunsdon, la toma de conciencia es aquella práctica o proceso mediante el cual se alcanza a entender que el ser mujer y sus experiencias se ubican en «un mundo de hombres», donde cada una habita las «estructuras de la feminidad» desde su individualidad sin que dicha experiencia personal escape de la mediación y el condicionamiento de su situación estructural en la sociedad como parte de un grupo oprimido. De esta forma, tal y como aclara Haraway (1995, p. 328), «cómo mirar desde abajo», esto es, cómo desarrollar, en el caso de las mujeres, una mirada consciente y críticamente feminista desde posiciones subyugadas, supone una cuestión extremadamente exigente en la medida en que 
no se aprende fácilmente y tampoco deja de acarrear problemas, incluso si «nosotras» habitamos «naturalmente» el gran terreno subterráneo de los conocimientos subyugados. Las posiciones de los subyugados no están exentas de re-examen crítico, de descodificación, de deconstrucción ni de interpretación, es decir, de los dos modos hermenéuticos y semiológicos de investigación crítica. Los puntos de vista de los subyugados no son posiciones «inocentes». (Haraway, 1995, p. 328)

Así, si entendemos el punto de vista como un visibilizador de un fenómeno simbólica y materialmente sepultado, como un «momento de percepción crítica» y feminista (Harding, 2004, p. 10), tal iluminación no surge inherente, automática ni espontáneamente por el mero hecho de pertenecer a una categoría social de base discriminatoria; por el contrario, el punto de vista constituye un logro posible, pero no certero, que debe ser alcanzado mediante la lucha inextricablemente política y científica y potencialmente emancipadora del propio grupo oprimido (Harding, 2004). De esta forma, como aclara Avtar Brah (2011, p. 153), «la proclamación de una identidad colectiva específica» constituye un «proceso político» mediante el cual cada grupo social se organizaría ideológica y operativamente en torno a sus objetivos; los «sectores dominantes» aspirarán a conservar y extender su potestad, mientras que los «sectores subalternos» batallarán por «renegociar o impugnar su situación» (Juliano, 2001, p. 15).

\subsection{Las tensiones de la identidad colectiva «mujeres»}

Retomando el párrafo anterior, el sentido social o colectivo de la identidad puede entenderse como un espacio hacia el cual conducen los distintos «senderos» demarcados por el conjunto de fuerzas e instituciones socioeconómicas y en el que convergen aquellas experiencias comunes y compartidas con otras personas en función, por ejemplo, del género, la clase o la raza (Hill Collins, 1997), esto es, en torno a un «eje específico de diferenciación» (Brah, 2011, p. 152). Este sentido social o colectivo es habitualmente movilizado, como en el caso de las TTFFPPV, en cuanto que «recurso simbólico» que politiza la diferenciación del grupo y posibilita su conmutación por derechos, reconocimiento, credibilidad, autoridad y visibilidad (Moran, 2015, pp. 47, 177). No obstante, esta politización estratégica implica, por una parte, interpretar la identidad social como una «propiedad sustantiva» del grupo 
y, por otra, instrumentalizarla como mecanismo delimitador y diferenciador (Moran, 2015, pp. 46-47). Para teóricas del punto de vista como Hill Collins (1997), el grupo social mujeres vendría sustentado por una localización común en las relaciones de poder, por experiencias históricamente compartidas y por «oportunidades y restricciones» similares, características que gozan de cierta «permanencia» en el tiempo y que trascienden las particularidades propias de las experiencias individuales de sus integrantes.

En este sentido, apelar a una identidad colectiva femenina desde las TTFFPPV parece comprensiblemente problemático, en línea con el cuestionamiento de una supuesta «unidad romántica» (Galindo, 2014, p. 54) o «vínculo común» (hooks, 2004, p. 37) o «natural» (Haraway, 1995, p. 264) entre las mujeres que, como sostiene Sylvia Walby (2001), reclama un sujeto comunitario sustentado por una noción de identidad más o menos estática que no permitiría (re)conocer la multiplicidad y solapamiento de las divisiones y desigualdades sociales ni la conceptualización del cambio y la fluidez. Precisamente, Hill Collins (1997) comprende que esta «fluidez» del colectivo mujeres no responde sino a las transformaciones de los mecanismos y estrategias de(l) poder que, a través de esos cambios, continúan reproduciendo desigualdades de género, clase y raza a través del tiempo, (re)estabilizando así los grupos sociales. Por tanto, la premisa de Hill Collins implica, por una parte, cierto determinismo social en línea con la negación de la agencia individual e incluso de la autogestión política y transformadora de los colectivos. Pero, siguiendo a hooks (2004, p. 37), el sexismo institucionalizado «nunca ha determinado de forma absoluta el destino de todas las mujeres de esta sociedad» como si de una «suerte común»se tratara.

Además, premisas como la de Hill Collins también reavivan inquietudes esencialistas no porque evoquen explícitamente una mujer ideal o mi(s)tificada, sino porque las identidades sociales con aspiración de permanencia se deben a cierto «consenso interno» (Walby, 2001) cuya normatividad entraña, en principio, diferencias excluyentes. Y es que la «marcación y ratificación de límites simbólicos, la producción de «efectos de frontera»», como explicaba Stuart Hall (2003, p. 16), es de suma importancia para las identidades colectivas, ya que no solo identifican lo común y compartido, sino que, además, señalan la alteridad, así como establecen las pautas relacionales con lo ajeno al grupo (Trujillo, 2009). De esta forma, sostiene Brah (2011, p. 
153), «lo común que se evoca solo puede tener significado en articulación con un discurso de la diferencia». La identificación de/con el grupo es un proceso que requiere de «lo que queda afuera, su exterior constitutivo» (Hall, 2003, p. 16); necesita, en definitiva, de esos «otros» a los que cerrar las puertas, excluir y herir (Zafra, 2004). Es por ello por lo que estas fronteras identitarias, añade Gracia Trujillo (2009), no están libres de conflicto, ya que fraccionan y dividen el propio interior del colectivo, generando belicosos debates sobre «quiénes somos y quiénes no somos».

Finalmente, en relación con las experiencias supuestamente compartidas por las mujeres que autoras como Hill Collins reclaman a modo de hilos que tejen su identidad colectiva, si bien es una premisa radicalmente veraz y valiosa debido al carácter estructural del patriarcado, esta merece algunas matizaciones. Para María Galindo (2014), Joanne Hollows (2000) y hooks (2004, 2017), las mujeres no comparten experiencias necesariamente idénticas en la medida en que son atravesadas por diferentes formas de opresión. De tal modo, la invocación de un sujeto femenino unitario resultaría para ellas sospechosa y no acabaría sino beneficiando a un grupo muy específico y privilegiado de mujeres (Oksala, 2014; Phipps, 2016; Trujillo, 2009). Como afirma Moya Lloyd (2005, pp. 22, 27), cartografiar las experiencias femeninas con la esperanza de descubrir «la verdad de la mujer» no genera sino «representaciones» de la feminidad que, si bien perseguirían una finalidad favorable, son, por otra parte, fácilmente cuestionables, además de evidenciar un desfase respecto a las realidades «plurales, multifacéticas y complejas» de las vidas de las mujeres.

\subsection{Los claroscuros del privilegio epistémico}

Diversos argumentos feministas han reiterado su preferencia por las posiciones subyugadas y las visiones «desde abajo» como una estrategia de justicia «para poder ver bien» (Haraway, 1995, pp. 327-328, 330). En las TTFFPPV, esta predilección se traduce en el supuesto privilegio epistémico de las mujeres (por ejemplo, Blázquez, 2011, 2012; Harding, 1996). Sus teóricas sostienen que son precisamente la desigualdad y la injusticia de sus condiciones de vida las que otorgan a las mujeres una óptica particular y diferenciada para reconocer la realidad social, de tal manera que dichas experiencias vitales 
en femenino son identificadas como epistemológicamente superiores o privilegiadas en la medida en que su posición subyugada posibilitaría un conocimiento de las relaciones, los fenómenos y las transformaciones de género «más completo y menos perverso» respecto al tradicionalmente generado desde posiciones masculinas de dominación (Blázquez, 2011, pp. 112-113, 2012, pp. 29-30; Harding, 1996, p. 24).

Sin embargo, la del privilegio epistémico resulta una premisa profundamente problemática, sensible, además, a multitud de críticas y recelos (por ejemplo, Haraway, 1995; Hekman, 1997; Walby, 2001), muy particularmente en torno a sus riesgos de automatización, homogeneización y esencialismo, de monopolio exclusivo para las mujeres y, en definitiva, de una aparente negación de la diversidad de los feminismos y de las propias mujeres (Harding, 1996). Desde el valor de estas críticas, podemos iluminar dos cuestiones centrales. Primero, y como sugiere la propia Harding (2004, p. 7), «iquiénes son estas «mujeres» cuyas experiencias, ubicaciones sociales y discursos son utilizados para fundamentar el conocimiento feminista?». Esto es, «¿qué identidades están disponibles para poner las bases de ese poderoso mito político llamado «nosotras»?» (Haraway, 1995, p. 264, 2016, p. 16); ¿lo personal de qué mujeres es más político, y por qué? (Phipps, 2016). $\mathrm{Y}$, en segundo lugar, ¿hasta qué punto, y en qué sentidos, los relatos de las mujeres explican de formas más preferibles y certeras la realidad social? Ambas cuestiones representan dos de los riesgos más «serios» de los que el privilegio epistémico, entendido por Haraway (1995, p. 328), adolece: el de «apropiarse» de las experiencias de las más desfavorecidas y el de «romantizar» la visión que posibilitan sus posiciones.

Para responder a la primera de las cuestiones, se antoja imprescindible el trabajo de Alison Phipps (2016) en torno a las politizaciones selectivas de las experiencias de las mujeres en el marco de la contrarrevolución neoliberal. Phipps explica que ciertos relatos son convenientemente priorizados, capitalizados y popularizados por los discursos hegemónicos para generar «empatías selectivas»y, por extensión, «ganancias políticas», reproduciendo así las dinámicas estructurales de poder en la medida en que invisibilizan deliberadamente otras narraciones y subjetividades. Esta lógica neoliberal, aclara Phipps, también habría infectado en cierto grado al propio feminismo, de tal forma que las «pugnas» feministas por «la «experiencia de las 
mujeres»» (Haraway, 1995, p. 183) privilegiarían, a juicio de Phipps (2016), determinados relatos personales en línea con «el uso estratégico de las narrativas victimizantes sobre mujeres» $\mathrm{y}$ «el racismo estructural del movimiento feminista», polarizando de esta forma los posicionamientos y dificultando la afinidad entre experiencias y relatos diversos.

En relación con la segunda de las cuestiones, cabe clarificar que el mero registro de aquello que las mujeres narran o creen resulta insuficiente para comprender nítidamente la vida social a partir de una investigación desde las TTFFPPV. Para Haraway (1995), los puntos de vista de los sujetos subyugados, como son en este caso las mujeres, suponen visiones preferentes en la medida en que proveerían conocimientos transformadores y más objetivos del mundo. Sin embargo, como también Haraway matiza, la mera condición o experiencia subyugada no constituye sustento alguno para una ontología, de tal manera que se nos antoja problemático -y puede añadirse, deshonesto, frívolo y de dudosa rigurosidad-privilegiar sistemáticamente como «expresiones fidedignas» las perspectivas de las mujeres, por mucho que dichas subjetividades y sus experiencias de vida radiquen en una condición de opresión (Harding, 2012, p. 51). Asimismo, siguiendo a Eli Bartra (2002, p. 154), esta preferencia por las visiones y experiencias de las mujeres no debe generar rechazo para con el resto de identidades, sino que debe entenderse como un posicionamiento de utilidad para «ver en dónde están [las mujeres] y qué hacen o no hacen y por qué»; como tampoco implica que, de forma apriorística y automática, todo aquello que habitan y hacen las mujeres sea «significativo y fundamental» per se.

\section{SOSTENIENDO AMBIVALENCIAS DESDE UNA PROPUESTA EPISTEMOLÓGICA CONCILIADORA: EL ENFOQUE POSMODERNO EN LAS TEORÍAS FEMINISTAS DE LOS PUNTOS DE VISTA}

¿Cómo sostener entonces las ambivalencias, tensiones y contradicciones de las TTFFPPV, así como la inevitabilidad esencialista de la identidad misma? Sugerimos para ello una propuesta desde la conciliación epistemológica de las TTFFPPV y el feminismo posmoderno que ponga en valor la tensión y la riqueza analítica del término medio entre ambas posturas y desafíe, además, 
discursos polarizados y lógicas maniqueas. Este proyecto conciliador no supone una posición acrítica, cobarde o equidistante; su aspiración a un término medio en tensión y profundamente feminista implica que el rechazo de cualquiera de las perspectivas moderna o posmoderna para siquiera comenzar a comprender cómo, quién(es), por qué y para qué somos aquí y ahora resulta sencillamente erróneo, falaz y deshonesto. Es por esto, explica Lloyd (2005, pp. 58-59), por lo que el feminismo «no puede ni debe» evitar el esencialismo modernista como tampoco debiera hacerlo con el sujeto-en-proceso posmoderno, quedando así, como entienden Genz y Benjamin Brabon (2009, p. 115), «suspendido entre su deseo de plantear un yo autónomo femenino/ feminista y la necesidad de tener que deconstruir el discurso moderno de la subjetividad».

La fluidez entre los posicionamientos epistemológicos del punto de vista y posmoderno, articulada por sus comprensiones comunes sobre la pluralidad de visiones y el carácter socialmente construido y contextual de la identidad (Hirschmann, 1998), habría contagiado la responsabilidad de diversidad de las teorizaciones posmodernas a las TTFFPPV (Blázquez, 2011, 2012), muy concretamente mediante el llamado sujeto posmoderno, que vendría a desmentir la existencia de ««la mujer» universal» o de ««la experiencia de la mujer»» en singular (Harding, 2002, p. 22). De esta forma, los nuevos feminismos posmodernos, posestructuralistas o de la tercera y cuarta ola (Gil, 2011) -entre los que destacan el transfeminismo, la teoría queer/cuir, los feminismos poscoloniales o los ciberfeminismos- se muestran escépticos respecto a las tendencias universalizantes del sujeto femenino y sus experiencias (Harding, 1996), renegando así del privilegio epistémico de las mujeres, reivindicado por las posiciones más convencionales de las TTFFPPV, frente a «la contingencia y la inestabilidad» de las identidades sociales (Blázquez, 2011 , p. 112; 2012, p. 29). De tal forma, este enfoque posmoderno afinaría la investigación de las llamadas identidades «fracturadas» (Haraway, 1995, p. 264), «fragmentadas» o «fragmentarias» (Harding, 1996, p. 142).

El primero de los síntomas a relucir de este fructífero encuentro epistemológico ha implicado asumir la pluralidad y la parcialidad de puntos de vista epistemológicamente relevantes de unas también plurales mujeres (Blázquez, 2011, 2012), permitiendo, en palabras de Nancy Hirschmann (1998), «el reconocimiento de la diferencia, la particularidad y el contexto» 
de sus experiencias materiales a través de la historia y de las relaciones de poder. Pero, además, añade Hirschmann, ha establecido «ciertos parámetros» a partir de los cuales considerar un punto de vista como feminista, entendiendo el feminismo en este sentido no como una noción «universal y atemporal», sino como «el producto de la negociación política continua dentro y entre los diversos grupos de mujeres que teorizan desde el punto de vista de sus experiencias de género, raza, clase y otras opresiones». A esta pluralización de las teorías, las visiones y las subjetividades vendría a sumarse igualmente la sofisticación del entendimiento de las identidades sociales, que reconocería la interseccionalidad de las vidas cotidianas desde la cual observar las relaciones de poder (Harding, 1997), así como (re) interpretaría dichos sujetos colectivos como construcciones sociales embrolladas, múltiples e históricamente situadas, en constante (re)elaboración y alteración (Brah, 2011; Hartsock, 1997). En definitiva, la identificación con un sujeto colectivo, explica Hall (2003), resultaría un proceso condicional y contingente, que viene además a complicarse en los últimos años, como sostiene Remedios Zafra (2004), por los movimientos migratorios, las diversas comunidades de «otros» que toman creciente protagonismo y la convivencia en internet.

No obstante, Harding (1996, p. 169) se pregunta si el encuentro de las TTFFPPV con el feminismo posmoderno posibilitaría una desvinculación suficiente de las primeras respecto a sus «antepasados modernistas» para producir explicaciones de la realidad social que evitaran la «peligrosa ficción» que para las epistemologías posmodernas supone «la «verdadera descripción» sobre nosotros mismos y el mundo que nos rodea» a la que sí parecen aspirar las epistemologías (modernas) del punto de vista. Esta es, sin duda, una pregunta de ida y vuelta que exige una contrapartida, y así lo entiende también Harding (1996, p. 26) cuando cuestiona, en términos similares, si podemos permitirnos renunciar a «una única descripción feminista y auténtica de la realidad», de inspiración claramente moderna, mientras la ciencia y «los proyectos sociales sexistas, clasistas, racistas e imperialistas» tejen profundas alianzas. Precisamente, esta «fragmentación infinita de perspectivas» posmodernas (Blázquez, 2011, p. 116, 2012, pp. 33-34), peligrosamente próxima al relativismo, la atomización y la descolectivización, es el principal impedimento para tejer «a priori» una cartografía común de

Feminismo/s 39, January 2022, 181-210 
luchas, intereses y objetivos en nombre de todas las mujeres (Gil, 2011, p. 36), de forma que el reto más delicado que enfrenta el giro posmoderno de las TTFFPPV es, en palabras de Norma Blázquez (2012, p. 34), «reconciliar las presiones de la diversidad y las diferencias con aquellas de la integración y la comunidad».

Así, la propuesta feminista de los puntos de vista, en los ojos (auto)críticos de Harding (como se cita en Blázquez, 2012, p. 44), «negocia continuamente e intenta redireccionar las tendencias más poderosas de los proyectos modernos y posmodernos». No podemos estar de acuerdo pues con Walby (2001) cuando esta afirma que la epistemología feminista, en la que se ubica la propuesta de las TTFFPPV, se ha retractado o retirado definitivamente del paradigma moderno para abrazar la parcialidad posmoderna de los conocimientos situados, especialmente cuando Hirschmann (1998), una de las hacedoras referentes de este abrazo epistemológico conciliador, reivindica el feminismo como un discurso que «es y debe ser en parte modernista»y en el que la noción de mujer es necesariamente fundacional, o cuando la propia Harding (1996, p. 169) reclama la necesidad de «los proyectos de ciencia sucesora» para la justa transformación de las relaciones sociales. Por tanto, desde una pretendida y consciente centralidad crítica en la hibridación epistemológica posmoderna y de los puntos de vista, tanto el «enfoque postmodernista del punto de vista» que recomienda Harding (1991, p. 49), como la propuesta teórica de los «Puntos de Vista Feministas Postmodernos» formulada por Hirschmann (1998) resultarían paradigmática y singularmente relevantes.

Pero ¿cómo salvar el que Hirschmann (1998) considera el mayor cisma entre sendas propuestas epistemológicas, esto es, la fractura entre la discursividad posmoderna y la «realidad material» de los puntos de vista? Para avenir ambas posturas en torno a una «realidad prediscursiva concreta»e inteligible para la discursividad posmoderna que, además, evite evocaciones esencialistas o estáticas, Hirschmann propone la herramienta reconciliadora del «momento materialista»: por un lado, avendría las maneras en las que las normas reguladoras «hacen realidad» las experiencias y condiciones concretas de vida de las mujeres con las posibilidades que la «realidad material» ofrece para aprehender sus experiencias cotidianas de desigualdad, articular reivindicaciones en torno a las mismas y, muy especialmente, «desafiar», 
contradecir y moldear los discursos mismos; y, por otra parte, precisamente desde este reconocimiento dual de las experiencias como discursivas y materiales, la crítica y la resistencia feministas cobrarían sentido y posibilidad, ofreciendo también una lectura mucho más compleja e informada de las opresiones que padecen las mujeres.

\section{CONCILIACIÓN EPISTEMOLÓGICA FEMINISTA: PUNTOS PRÁCTICOS DE SUTURA}

\subsection{Enfrentar el relato único sobre las mujeres a partir de sus diversas experiencias}

Las experiencias de las mujeres suponen recursos de gran valor científico en la medida en que constituyen expresiones elocuentes y relevantes de «la realidad» a estudiar (Bartra, 2002; Harding, 2002). En este marco, como explica Harding (1996, p. 24), el feminismo proporciona la teoría y el estímulo investigador y político para convertir la perspectiva y las experiencias de estas mujeres en un «punto de vista», esto es, en un «fundamento moral y científicamente preferible»-que no automáticamente privilegiado ni verdadero- para interpretar y explicar la vida en sociedad. La predilección de las TTFFPPV por las experiencias y relatos femeninos rearticularía la subjetividad científica de las mujeres, representándolas no como objetos de estudio estáticos y eternos, sino como sujetas activamente implicadas en procesos socioeconómicos orgánicos (Pérez-Orozco, 2014), esto es, como sujetas «de conocimiento» (Blázquez, 2011, p. 109; Harding, 2002, p. 14). Este (re) conocimiento, además, posibilitaría investigaciones feministas «a favor de las mujeres», frente a la tradicional predilección masculina de la ciencia, de forma que se producirían conocimientos para ellas y sus (pre)ocupaciones en lugar de para las instituciones dominantes (Harding, 2002, pp. 23-24).

Asimismo, valorar científicamente las experiencias de las mujeres haría florecer la apreciación de sus (muchos) relatos y vivencias frente a los peligros de lo que Chimamanda Ngozi Adichie (2009) nombra como «el relato único». Las experiencias femeninas pueden ser «normalizadas, empobrecidas, dolorosas, degradantes o desempoderantes» (Oksala, 2014) y sus relatos, además, pueden movilizarse desde posiciones hegemónicas para «desposeer 
y dañar» a sus protagonistas, generando un retrato unívoco y estereotípico que acaba por convertirse en profecía autocumplida (Adichie, 2009). Así vendría a actuar, por ejemplo, el discurso universalista de «la «experiencia femenina»» (Haraway, 1995, p. 190; Phipps, 2016). Pero las experiencias de las mujeres, al igual que sus relatos, también pueden ser «reconstruida[s], recordada[s], rearticulada[s]» (Haraway, 1995, p. 190), encerrando así la potencialidad de empoderar, humanizar y dignificar a las personas implicadas en su narración y vivencia (Adichie, 2009), frente al tradicional obscurecimiento de las experiencias femeninas indefectiblemente vinculadas a una cotidianeidad despreciada y a la otredad respecto a la experiencia universal masculina (Brunsdon, 2007).

\subsection{Partir de las experiencias encarnadas para articularlas en contexto}

Como sostiene Harding (2012, pp. 50-51), las TTFFPPV deben estudiar "hacia arriba», motivadas no por la mera narración de las condiciones de vida de las mujeres, sino por la ambición de trazar, a partir de las mismas y desde la teoría crítica, «el mapa de las prácticas del poder, de las maneras en que las instituciones dominantes y sus marcos conceptuales crean y mantienen relaciones sociales opresivas». Se trata, pues, de articular «afinidades» a través del mar de puntos de vista femeninos (Haraway, 1995, p. 191), para alcanzar así sus dimensiones culturales, políticas y económicas, esto es, para «descubrir lo social» (Smith, 1997).

No obstante, en un constante transitar de ida y vuelta entre lo local y lo global, lo individual y lo colectivo (Esteban, 2004), las experiencias y relatos de las mujeres no solo tienen un valor instrumental para comprender la arquitectura de poderes que las sostienen desde arriba. Además de ser valiosas en sí y por sí mismas, estas vivencias y narraciones constituirían también ejemplos de (re)construcción, subversión y/o negociación de los discursos y la materialidad de las macroestructuras socioeconómicas y sus instituciones, ofreciendo así la oportunidad de desarrollar un análisis multinivel que parta de estas experiencias en contexto. En este sentido, reconoce Harding (como se cita en Hirschmann, 1998), las TTFFPPV deben teorizar críticamente sobre y a partir de las experiencias de las mujeres para comprender en qué formas y sentidos estas responden a sus opresiones. Este objetivo en ningún 
caso implica afirmar que las mujeres, desde su individualidad más o menos privilegiada o en colectivo, sean capaces de zafarse a voluntad de dichas opresiones, sino que viene a sostener, a nuestro entender y como también afirman Marchand y Runyan (2011), que el abanico de reacciones y actitudes que las mujeres encarnan frente a sus experiencias de (des)igualdad no demuestra sino su subjetivación como seres agentes.

De cualquier otra forma, un análisis de la experiencia hipersituado, aislado y ajeno a lo estructural no solo dinamita el compromiso político empoderante y emancipador de las TTFFPPV, sino que, además, reproduce la lógica individualista del neoliberalismo, que enajena dichas experiencias de sus contextos socioeconómicos y entramados de poder para incorporarlas a un escenario mercantilizado y altamente competitivo donde se despliega una ilusión igualitaria que reitera la ventaja política y mediática de las personas más privilegiadas a la par que encubre su poder estructural (Phipps, 2016). Desgraciadamente, como sostiene Hill Collins (1997), la minuciosa atención que merecen las cuestiones de agencia individual y diversidad parece haber oscurecido en cierta medida la importancia de las experiencias colectivas o compartidas en favor de un individualismo civilmente empoderante (desde la perspectiva del liberalismo burgués), celebradamente diferenciador (según la teoría posmoderna) y falsamente liberador (para la ideología de libre mercado). Esta reflexión se antoja de inigualable valor en un contexto socioeconómico y cultural como el actual, en el cual la fetichización de la libre elección hiperindividualista, el autoempoderamiento y la diversidad estética estimulada por el «posfeminismo neoliberal» (Martínez-Jiménez, 2021) oscurece el análisis estructural de las relaciones de poder y resignifica las desigualdades como obstáculos personales y anecdóticos motivados por malas decisiones, de forma tal que su superación, al igual que su culpa, suponen una responsabilidad individual y privativa. Es en este sentido que autoras como Joan Scott (como se cita en Phipps, 2016) desconfían profundamente de «la autoridad epistemológica de la experiencia» en la investigación feminista ya que, si bien «el giro hacia la experiencia» visibiliza una gran diversidad de relatos y opresiones vividas, para Scott tomar la experiencia como punto de partida no tiende sino a reificar las narraciones personales como explicaciones deshistorizadas que, además, esencializan 
las identidades y pueden terminar por apuntalar, en lugar de contestar, los sistemas ideológicos.

Sin duda, Scott apunta un riesgo manifiesto para cualquier estudio irresponsable, relativista o negligente basado en la experiencia, pero no, creemos, para proyectos feministas críticos. En contra de lo sugerido por Scott, las necesarias cautelas en torno al individualismo neoliberal que acecha la producción académica no deberían impedirnos teorizar desde y sobre la experiencia personal, ya que esta constituye «un producto y un medio importantísimo» para los feminismos, que deben «luchar por los términos de su articulación» (Haraway, 1995, p. 184) y, como señala Johanna Oksala (2014), «recuperar» críticamente una experiencia alejada de esencialismos y generalizaciones con el ánimo y la posibilidad de disputar así «identidades, normas y esquemas conceptuales», generar «expresiones culturales» más ricas y forjar solidaridades transversales. Por tanto, siguiendo a Judith Butler (como se cita en Soley-Beltran y Preciado, 2007), la primera persona puede ser un valioso "punto de partida» para comprender, a partir de sus experiencias, cómo «una serie de normas sociales o restricciones o condiciones de agencia» nos ubican diferencialmente en el entramado de relaciones de poder.

Pero, como también advierte Butler (como se cita en Soley-Beltran y Preciado, 2007), el yo debe «des-orientar[se]», superarse, para integrarlo en «esas condiciones más amplias» que lo posibilitan. En este sentido, las políticas de lo personal o de la primera persona resultan centrales para comprender las subordinaciones y explotaciones de las mujeres en sus propias palabras y experiencias, pero estas deben trascenderse y ubicarse en un análisis estructural (Brunsdon, 2007; Phipps, 2016), sorteando así el peligro de «queda[r] atrapado» tanto en el embelesamiento y la descontextualización de la primera persona sobre el que nos previene Butler (como se cita en Soley-Beltran y Preciado, 2007), como en la mercantilización neoliberal de las narrativas personales (Phipps, 2016); y, muy especialmente, manteniendo «la necesaria articulación de la experiencia femenina [...] con la lucha por comprender los determinantes de esta experiencia» para, así, transformarla en un sentido que supere la individualidad (Brunsdon, 2007, p. 496). 


\subsection{Abandonar el privilegio epistémico por la preferencia crítica}

Frente al problemático mecanismo del privilegio epistémico, Phipps (2016) propone reconocer la validez de todas las experiencias femeninas a pesar de encontrarse «asimétricamente situadas». En la medida en que, como sugiere Brunsdon (2007), este reconocimiento aprehenda las desigualdades que encarnan las mujeres más allá del sexismo, la iluminación de estas experiencias asimétricas permitiría escapar de la trampa original que implica el conocimiento a través de las mismas y que reificaría un sujeto unitario en torno a una opresión común. La generación de una conciencia feminista, explica así Brunsdon (2007, p. 496), es una tarea que debe asumir las diferentes opresiones que padecen «las mujeres negras, las lesbianas, las mujeres mayores, las mujeres de clase trabajadora, así como aquellas mujeres más articuladas dentro de la cultura dominante, jóvenes, heterosexuales, blancas de clase media».

No obstante, el reconocimiento de estas diferencias intragénero podría conducirnos, como coligen Harding (1996), Hill Collins (1997) y Chandra Talpade Mohanty (2008), a pensar en los puntos de vista de las mujeres negras/racializadas/del Sur global como más auténticos y privilegiados para el conocimiento de la realidad social, en la medida en que no han participado de la dominación sexista, racista y clasista como sí lo hacen las mujeres blancas, ofreciendo de esta forma «la visión más inclusiva del poder sistémico» (Mohanty, 2008, pp. 421-422). En este sentido, seguimos a Hirschmann (1998) y Pérez-Orozco (2014) cuando estas matizan acertadamente que la multiplicidad, la interdependencia y la transversalidad de los ejes de opresión -y su propia vivencia inextricable, fluctuante y contingente, podría añadirse-generan puntos de vista subyugados diversos, inestables e, incluso, contradictorios, impidiendo así nombrar «un mítico sujeto oprimido homogéneo cuya óptica sea preferible» (Pérez-Orozco, 2014, p. 69). De la misma manera, consideramos, sería poco riguroso instrumentalizar identidades aglutinadoras y definitivas que representen a las mujeres blancas como un todo acrítico, constante y cómplice cuyas experiencias de marginalidad y opresión, si bien aliviadas y/o ambivalentemente sofisticadas por su condición comparativa de privilegio, deban ser ignoradas o silenciadas, tal y como niegan Hirschmann (1998), Hollows (2000) o Brunsdon (2007). 
En este marco investigador, la preferencia crítica por las experiencias y los relatos de las mujeres responde a un criterio no puramente epistemológico, sino, muy especialmente, «ético y político» (Hartsock, 1997). Para comprender los aristados planteamientos y experiencias que relatan las mujeres sobre/en «esa Cosa escandalosa» con la que Haraway (1995, p. 340) alude al «"patriarcado capitalista blanco"», se antoja necesario un análisis desde las TTFFPPV que, por un lado, no desdeñe en cuanto que equívocas o envenenadas las perspectivas femeninas no reconocidas en el feminismo, sino que contextualice su desidentificación con los feminismos, y su potencial complicidad patriarcal, en su debido escenario socioeconómico, político-ideológico y cultural. Como afirma Oksala (2014), los «relatos experienciales» de las mujeres sobre el mundo que habitan tienen potencial de veracidad, pero dicho potencial también cobija la posibilidad de relatos erráticos o, si se prefiere, incompletos o viciados. Reconocerlo no implica, sin embargo, que dichas narraciones sean «insignificantes o necesariamente falsas», sino desmitificar una noción idealizada de la experiencia femenina como «epistémicamente infalible». En este sentido, las palabras de Helen Davis (2004, p. 152) cobran especial valor al reclamar la necesidad de «observar aquello que las personas experimentan como verdadero y significativo», en lugar de focalizar los esfuerzos en identificar la «falsedad» de la «experiencia ideológica».

Además, esta radiografía desde las TTFFPPV registraría al mismo tiempo la posibilidad (y deseabilidad) de que estas mujeres que no se identifican como feministas trasciendan sus puntos de vista femeninos para llegar a reconocerse en el feminismo desde una subjetividad en constante proceso, en la medida en que, como también señala Oksala (2014), estamos inmersas en un proceso incesante de (re)ajuste y negociación de nuestro pensamiento a partir de nuestras experiencias y viceversa, pudiendo derivar en una toma de conciencia y esta, a su vez, en prácticas críticas y feministas de (auto) transformación y acción política colectiva.

Con todo, este asunto se complica hasta el punto de cuestionar si es entonces potestad legítima de las investigadoras/pensadoras decidir quién es feminista y quién no lo es, entrando así en juego cierta «autoridad»o «vanguardia» feminista científica (Hollows, 2000, pp. 203, 35), entendida por Brunsdon (como se cita en Hollows, 2000, p. 35) como una suerte de «identidad feminista políticamente correcta» en oposición al resto de «mujeres 
ordinarias» construidas como otredades «inválidas» a las que señalar sus errores. Con todo, la problematización de esa autoridad feminista que señalaba Hollows -autoridad que, por otra parte, cimienta la valiosa diferencia de Hartsock entre puntos de vista femeninos y feministas- no significa que las investigadoras se inhiban de analizar y comentar, desde su formación teórico-académica y su compromiso crítico feminista, los relatos de las mujeres en relación a su identificación o desapego para con el feminismo, en la medida en que, como sostiene Scott (como se cita en Hollows, 2000, p. 9), la experiencia no constituye per se una explicación o evidencia incontestable de la realidad social, sino que «es a la vez una interpretación y necesita una interpretación».

\section{COMENTARIOS FINALES: SOBRE LA RESPONSABILIDAD DE LA INVESTIGADORA EN LA EPISTEMOLOGÍA FEMINISTA CONCILIADORA}

Harding (2002, p. 24) reclama la figura de la propia investigadora como «nuevo objeto de investigación» que debe ser situado «en el mismo plano crítico que el objeto explícito de estudio», de tal forma que la supuesta brecha desvinculante entre la investigadora o sujeto conocedor y las mujeres-objeto a conocer es puesta en evidencia (Blázquez, 2011, 2012; Esteban, 2004; PérezOrozco, 2014). En este sentido, las investigaciones feministas reconocerían la intersubjetividad propia del proceso científico y calificarían esta relación entre investigadoras y sujetos de estudio como (auto)reflexividad (Harding, 2002).

Esto no quiere decir, como bien sugiere Hall (como se cita en Morley y Chen, 2005, p. 402), que las ideas y la teorización no sean posibles más allá de la propia experiencia, ni que las investigadoras deban relajar sus precauciones en relación a la «peculiar forma» que toma su existencia individual. En otras palabras: «todo científico social tiene su subjetividad, reconoce Arlie Russell Hochschild (2008, p. 18), la cuestión es determinar cómo la usa». Precisamente, desde una plena consciencia de la responsabilidad que implica la puesta en valor de lo subjetivo, Hall insiste en sus recelos sobre someter a una (auto)crítica suficiente y profunda la propia experiencia cuando esta 
sirve de base para el conocimiento sin contemplar, además, otras experiencias no habitadas personalmente (como se cita en Morley y Chen, 2005, p. 402). No obstante, lejos de limitarse a una producción teórica autocomplaciente o a un ejercicio vacuo y ensimismado de enunciación a la manera del individualismo neoliberal despolitizado (Biglia, 2015; Esteban, 2004; PérezOrozco, 2014), la (auto)reflexividad -esto es, el yo como «instrumento de investigación» (Hochschild, 2008, p. 17)-implica localizarse a una misma en un sentido relacional y estructural (Braidotti, 2011); es decir, explicitarse como un sujeto «real, histórico, con deseos e intereses particulares y específicos» (Harding, 2002, p. 25), para practicar desde ahí la «problematización» crítica de la propia experiencia (Oksala, 2014) y responsabilizarse de los sesgos propios en la producción de saberes (Biglia, 2014; Braidotti, 2011; Haraway, 1995; Harding, 2002; Pérez-Orozco, 2014).

Como reconoce Hall, «las identidades de las personas están ligadas a su posicionamiento intelectual» (como se cita en Morley y Chen, 2005, p. 401). Precisamente en esta línea no son pocas las autoras que reclaman la relevancia de la identidad de género de la investigadora, al igual que su determinante autoidentificación, experiencia y activismo feministas, como una sensibilidad que diferencia -o debería diferenciar- la investigación (Bartra, 2002); pero, sobre todo, como un compromiso cotidiano ineludible para producir conocimientos libres de distorsiones sexistas y androcéntricas (Harding, 2002). Por tanto, las investigaciones y las investigadoras feministas exigirían una «autoreflexividad radical-un cuestionamiento reflexivo de la propia experiencia» (Oksala, 2014), entendiendo que la propia investigadora encarna, en mayor o menor medida, dicha subordinación.

De tal manera, lo que Mari Luz Esteban (2004) nombra como «la influencia de lo vivido sobre lo escrito» otorga un segundo sentido a ese resituar a la investigadora en el mismo plano crítico que sus sujetos de estudio: el sentido del embodiment o la «encarnación conflictual, interactiva y resistente de los ideales sociales y culturales» (Esteban, 2004). Así, «hablar de y desde una/o misma/o, partir de la propia experiencia, de las propias contradicciones, conflictos y placeres, no despojarse del propio cuerpo, ni censurarlo» (Esteban, 2004) -esto es, habitar el asunto mismo sobre el que se reflexiona y escribe- supone a todas luces, como reconoce Susan Bordo (2008), una complicación añadida a la investigación. Pero, asumida esta complicación,

Feminismo/s 39, January 2022, 181-210 
y muy especialmente para los feminismos, las investigaciones encarnadas no deberían identificarse de forma sistemática como prácticas laxas, poco rigurosas o incluso ilegítimas (Hochschild, 2008; Oksala, 2014). Como sostiene Hochschild (2008, p. 18), necesitamos y debemos «poner a prueba nuestros pálpitos de múltiples maneras» $\mathrm{y}$ «cuestionar nuestros valores continuamente», pero tal posicionamiento (auto)crítico no debería suponer la renuncia a la orientación que dichos pálpitos y valores pueden ofrecer en el ejercicio científico.

Resulta ineludible, pues, mirarse a una misma en tanto investigadora situada, por tanto, desde cierto privilegio académico de partida, para revisar así la responsabilidad nada inocente que implica seleccionar e interpretar las experiencias, voces y puntos de vista de las mujeres. Como afirma Phipps (2016), en el «mercado de la experiencia»-y podemos añadir, en una academia intensamente neoliberalizada- parece «inevitable» que las voces y narraciones más privilegiadas gocen de un eco mayor, incluido el análisis que la propia investigadora elabora a partir de las experiencias de determinadas mujeres y que, por extensión, como señala Linda Alcoff, encierra asimismo el peligro de «hablar por las demás» (como se cita en Phipps, 2016). Acerca de este peligro, vale la pena reproducir las palabras de Barbara Biglia (2015) en torno a la crítica feminista a la representatividad:

[...] aun estudiando colectividades a las que pertenecemos, no podemos eximirnos de estar, de alguna manera, representando a subjetividades y/o colectividades específicas. Quizá por ello deberíamos reconocer que cuando producimos conocimientos, siempre estamos «representando» realidades y sujetos. Por lo tanto, tenemos que hacernos cargo de lo que implica representar, asumir las responsabilidades que comporta, en lugar de atrincherarnos en la negación de esta práctica. No se trata de volver a hablar en nombre de otras y ningunear así a las subjetividades y colectividades minorizadas, sino de reconocer que cuando asumimos el rol de altavoces de las palabras de los sujetos con los que investigamos, no estamos exentas de modificar su mensaje. (pp. 32-33)

Así, si bien el énfasis crítico en el valor político de lo personal y subjetivo representa una oportunidad para romper con los lenguajes arcaicos de la objetividad que vienen colonizando la actividad intelectual, la visión y la visibilidad (científicas), tal y como afirma Haraway (1995, p. 330), son «siempre una cuestión del «poder de ver»y, quizás, de la violencia implícita en 
nuestras prácticas visualizadoras» que exige, por tanto, un compromiso constante para sostener una mirada investigadora enriquecida, honesta y fundamentada que no niega ni resuelve en falso los conflictos que habita. A este compromiso, precisamente, quiere contribuir la propuesta práctica de una epistemología feminista conciliadora que defiende el presente trabajo.

A través de este tenso y fértil diálogo entre los distintos marcos epistemológicos feministas (especialmente, los de herencia moderna y los posmodernos), este texto quiere animar a las investigaciones e investigadoras feministas, en un momento particularmente delicado para nuestra conversación pública, a transitar los actuales debates en torno a la (in)validez de categorías convencionales como género y mujer o a la (i)legitimidad de unas voces y experiencias respecto a otras desde la responsabilidad crítica de cuidar-nos y reconocer-nos en nuestra innegable diversidad, explorando al mismo tiempo, en palabras de Silvia L. Gil (2011), las posibilidades de tramar «sentidos comunes en la dispersión». Desde este compromiso conciliador también queremos huir de caricaturas simplistas de sendas orillas moderna y posmoderna del feminismo - de la supuesta rigidez esencialista y el agotamiento de la primera, y de la irresponsabilidad relativista y antimaterialista achacada a la última-, así como del dilema impostado entre entregarse o renunciar definitiva y absolutamente a una de ellas, alimentado por lógicas dicotómicas que los propios feminismos críticos tanto se empeñan en denunciar. Defendemos, por tanto, que a través de esta conciliación práctica, que no pretende ser naíf ni sepultar discordias (incluso violencias ejercidas en nombre de la teoría sobre las vidas de mujeres negadas y vulnerabilizadas), podríamos encaminarnos hacia la generación de «saberes transformadores» (Biglia, 2015, p. 21) con los que poder elaborar, en palabras de Haraway (1995, pp. 338, 321), «mejores versiones del mundo» para «vivir bien en él».

\section{REFERENCIAS BIBLIOGRÁFICAS}

Adichie, C. N. (2009, julio). Chimamanda Ngozi Adichie: The danger of a single story [Vídeo]. TED. https://www.ted.com/talks/chimamanda_ adichie_the_danger_of_a_single_story

Bartra, E. (2002). Reflexiones metodológicas. En E. Bartra (Comp.), Debates en torno a una metodología feminista (pp. 141-158). Universidad Autónoma Metropolitana y Universidad Nacional Autónoma de México. 
Biglia, B. (2015). Avances, dilemas y retos de las epistemologías feministas en la investigación social. En I. Mendia, M. Luxán, M. Legarreta, G. Guzmán, I. Zirion y J. Azpiazu (Eds.), Otras formas de (re)conocer: Reflexiones, herramientas y aplicaciones desde la investigación feminista (pp. 21-44). Hegoa.

Blázquez, N. (2011). El retorno de las brujas: Incorporación, aportaciones y críticas de las mujeres a la ciencia. Universidad Nacional Autónoma de México.

Blázquez, N. (2012). Epistemología feminista: Temas centrales. En N. Blázquez, F. Flores y M. Ríos (Coords.), Investigación feminista: Epistemología, metodología y representaciones sociales (pp. 21-38). Universidad Nacional Autónoma de México.

Bordo, S. (2008). Cassie's hair. En S. Alaimo y S. Hekman (Eds.), Material feminisms (pp. 400-424). Indiana University Press.

Brah, A. (2011). Cartografías de la diáspora. Identidades en cuestión. Traficantes de sueños.

Braidotti, R. (2011). Nomadic subjects: Embodiment and sexual difference in contemporary feminist theory. Columbia University Press.

Brunsdon, C. (2007). 'It is well known that by nature women are inclined to be rather personal'. En A. Gray, J. Campbell, M. Erickson, S. Hanson y H. Wood (Eds.), CCCS Selected working papers (Vol. 2, pp. 485-499). Routledge.

Davis, H. (2004). Understanding Stuart Hall. Sage.

Esteban, M. L. (2004). Antropología encarnada. Antropología desde una misma. Papeles del CEIC, 12, 1-21. http://www.ehu.eus/ojs/index.php/papelesCEIC/ article/view/12093/11015

Galindo, M. (2014). ¡A Despatriarcar! Feminismo Urgente. Lavaca.

Gálvez, L. (2017, 26 de enero). ¿El Neoliberalismo produce una Democracia sobrante? [Vídeo]. Vimeo. https://vimeo.com/209284569

Genz, S. (2009). Postfemininities in popular culture. Palgrave Macmillan. https:// doi.org/10.1057/9780230234413

Genz, S. y Brabon, B. A. (2009). Postfemininism. Cultural texts and theories. Edinburgh University Press.

Gil, S. L. (2011). Nuevos Feminismos. Sentidos comunes en la dispersión. Una historia de trayectorias y rupturas en el Estado español. Traficantes de Sueños.

Gill, S. y Roberts, A. (2011). Macroeconomic governance, gendered inequality, and global crises. En B. Young, I. Bakker y D. Elson (Eds.), Questioning financial governance from a feminist perspective (pp. 155-172). Routledge. 
Hall, S. (2003). Introducción: ¿quién necesita «identidad»? En S. Hall y P. du Gay (Comps.), Cuestiones de identidad cultural (pp. 13-39). Amorrortu Editores. Haraway, D. J. (1995). Ciencia, cyborgs y mujeres: La reinvención de la naturaleza. Cátedra.

Harding, S. (1991). Whose science? Whose knowledge? Thinking from women's lives. Cornell University Press.

Harding, S. (1996). Ciencia y feminismo. Ediciones Morata.

Harding, S. (2002). ¿Existe un método feminista? En E. Bartra (Comp.), Debates en torno a una metodología feminista (pp. 9-34). Universidad Autónoma Metropolitana y Universidad Nacional Autónoma de México.

Harding, S. (2004). Introduction: Standpoint theory as a site of political, philosophic, and scientific debate. En S. Harding (Ed.), The feminist standpoint theory reader: Intellectual and political controversies (pp. 1-16). Routledge.

Harding, S. (2012). ¿Una filosofía de la ciencia socialmente relevante? Argumentos en torno a la controversia sobre el Punto de vista feminista. En N. Blázquez, F. Flores y M. Ríos (Coords.), Investigación feminista: Epistemología, metodología y representaciones sociales (pp. 39-66). Universidad Nacional Autónoma de México.

Hartsock, N. C. M. (1997). Comment on Hekman's «Truth and method: Feminist standpoint theory revisited»: Truth or justice? Signs, 22(2), 367-374. https://doi.org/10.1086/495161

Hekman, S. (1997). Truth and method: Feminist Standpoint Theory revisited. Signs, 22(2), 341-365. https://doi.org/10.1086/495159

Hernando, A. (2012). La fantasía de la individualidad. Sobre la construcción sociohistórica del sujeto moderno. Katz Editores. https://doi.org/10.2307/j. ctvm7bdns

Hill Collins, P. (1997). Comment on Hekman's «Truth and method: Feminist standpoint theory revisited»: Where's the power? Signs, 22(2), 375-381. https://doi.org/10.1086/495162

Hirschmann, N. J. (1998). Feminist Standpoint as Postmodern Strategy. Women E Politics, 18(3), 73-92. https://doi.org/10.1300/J014v18n03_05

Hochschild, A. R. (2008). La mercantilización de la vida intima: Apuntes de la casa y el trabajo. Katz.

Hollows, J. (2000). Feminism, femininity and popular culture. Manchester University Press. 
Hooks, B. (2004). Mujeres negras. Dar forma a la teoría feminista. En b. hooks, A. Brah, C. Sandoval, G. Anzaldúa, A. Levins, K-K. Bhavnani, C. T. Mohanty (Autoras), Otras inapropiables: Feminismos desde las fronteras (pp. 33-50). Traficantes de Sueños.

Hooks, B. (2017). El feminismo es para todo el mundo. Traficantes de Sueños.

Juliano, D. (2001). El juego de las astucias. Mujeres y construcción de modelos sociales alternativos. Horas y HORAS.

Lloyd, M. (2005). Beyond identity politics: Feminism, power and politics. Sage.

Marchand, M. H. y Runyan, A. S. (2011). Gender and global restructuring: Sightings, sites and resistances. Routledge. https://doi.org/10.4324/9780203894972

Martínez-Jiménez, L. (2021). Postfeminismo neoliberal: una propuesta de (re) conceptualización desde los estudios culturales feministas. Investigaciones Feministas, 12(2), 371-382. https://doi.org/10.5209/infe.73049

Martínez-Jiménez, L., Gálvez-Muñoz, L. y Solano-Caballero, Á. (2018). Neoliberalism goes pop and purple: Postfeminist empowerment from Beyoncé to Mad Max. The Journal of Popular Culture, 51(2), 399-420. https:// doi.org/10.1111/jpcu.12671

Mohanty, C. T. (2008). De vuelta a «Bajo los ojos de Occidente». La solidaridad feminista a través de las luchas anticapitalistas. En L. Suárez y R. A. Hernández (Eds.), Descolonizando el feminismo: teorías y prácticas desde los márgenes (pp. 407-464). Cátedra.

Moran, M. (2015). Identity and capitalism. Sage. https://doi. org/10.4135/9781473910560

Morley, D. y Chen, K-H. (2005). Stuart Hall. Critical dialogues in Cultural Studies. Routledge.

Nicholson, L. J. (Comp.) (1992). Feminismo/posmodernismo. Feminaria Editora.

Oksala, J. (2014). In defense of experience. Hypatia, 29(2), 388-403. https://doi. org/10.1111/hypa.12053

Pérez-Orozco, A. (2014). Subversión feminista de la economía. Aportes para un debate sobre el conflicto capital vida. Traficantes de sueños.

Phipps, A. (2016). Whose personal is more political? Experience in contemporary feminist politics. Feminist Theory, 17(3), 303-321. https://doi. org/10.1177/1464700116663831

Sayer, A. (2009). Who's afraid of Critical Social Science? Current Sociology, 57(6), 767-786. https://doi.org/10.1177/0011392109342205 
Smith, D. E. (1997). Comment on Hekman's «Truth and Method: Feminist Standpoint Theory Revisited». Signs, 22(2), 392-398. https://doi. org/10.1086/495164

Soley-Beltran, P. y Preciado, B. (2007). Abrir posibilidades. Una conversación con Judith Butler. Entrevista a Judith Butler por Patrícia Soley-Beltran y Beatriz Preciado. Lectora, 13, 217-239. http://revistes.ub.edu/index.php/ lectora/article/view/7411

Trujillo, G. (2009). Del sujeto político la Mujer a la agencia de las (otras) mujeres: El impacto de la crítica queer en el feminismo del Estado español. Política y Sociedad, 46(1-2), 161-172. http://revistas.ucm.es/index.php/POSO/article/ view/POSO0909130161A/21882

Walby, S. (2001). Against epistemological chasms: The science question in feminism revisited. Signs, 26(2), 485-509. https://doi.org/10.1086/495601

Zafra, R. (2004). E-identidades: Loading-searching-doing (Cartografías del sujeto online). Remedioszafra.net. Recuperado el 5 de octubre de 2021. http://www.remedioszafra.net/edentidades/ 